\title{
Can Axillary Lymph Node Dissection Be Safely Omitted for Early-Stage Breast Cancer Patients with Sentinel Lymph Node Micrometastasis?
}

\author{
Sameer Damle, MD and Christine B. Teal, MD, FACS \\ Department of Surgery, Breast Care Center, The George Washington University Medical Center, Washington, DC
}

This study presents data that we have been anxiously awaiting. It adds to a slowly growing body of evidence that axillary lymph node dissection (ALND) may be omitted in select groups of node-positive patients. Many of us prefer not to perform ALND when only micrometastases are identified in sentinel lymph nodes (SLNs), especially when it often involves returning to the operating room. It is already the standard of care to omit ALND for patients who have SLNs positive only by immunohistochemistry (IHC). There are now tools available, such as the Memorial SloanKettering nomogram, that use tumor size, grade, number of positive SLNs, method of detection, estrogen status, and presence of lymphovascular invasion to determine the risk of finding additional positive axillary nodes if ALND is performed. ${ }^{1}$ Numerous studies have demonstrated a worse prognosis associated with axillary micrometastasis. ${ }^{2-5}$ However, these studies are retrospective, and some evaluated ALNDs performed before the era of routine SLN biopsies. What is most interesting about this study is that it prospectively evaluated the omission of ALND for micrometastasis, and it has the longest follow-up available in the literature for such patients.

Langer and colleagues demonstrate that patients with tumors of $<3 \mathrm{~cm}$ with micrometastatic foci $0.2-2 \mathrm{~mm}$ on hematoxylin and eosin (H\&E) stain or IHC have no worse 5-year survival than their node-negative counterparts, despite the omission of a completion ALND. The nodepositive patients did not have axillary recurrences or distant metastasis over a follow-up period ranging from 24-

(C) Indian Association of Surgical Oncology 2011

Published Online: 25 March 2011

This article was originally published in Annals of Surgical Oncology, volume 16, pp. 3215-3216, DOI 10.1245/s10434-009-0702-3.

C. B. Teal, MD, FACS

e-mail: cteal@mfa.gwu.edu
106 months. From these data, the authors conclude that ALND may be safely omitted in early stage breast cancer patients with SLN micrometastasis. The conclusion that ALND may be omitted in all early stage patients with micrometastases, including those with high-grade tumors $>2 \mathrm{~cm}$ and with lymphovascular invasion, is perhaps premature on the basis of their low numbers and short follow-up. The American College of Surgeons Oncology Group (ACOSOG) Z0010 and Z0011 studies will provide more answers with larger numbers and longer follow up.

It is interesting to note that the authors found a 5-year disease-free survival advantage in patients who were found to have micrometastatic disease over node-negative patients. Colpaert and colleagues found that the primary tumor was an independent prognostic factor while axillary micrometastasis were not, and they proposed that micrometastatic disease somehow primes the immune system, allowing for a more robust response against further tumor invasion. ${ }^{6}$ The 5 -year overall survival in the study by Langer and colleagues was equal in both groups.

The authors' rate of finding micrometastasis in $12 \%$ of their patients is consistent with the existing literature. ${ }^{7}$ However, this yielded only 27 patients, and approximately half of these patients were positive by IHC. The authors do not clarify whether the micrometastasis in the 13 patients identified by IHC were subsequently identified by H\&E, which may affect their statistical analysis. Currently, many surgeons will perform a completion ALND for micrometastases found by H\&E, but not by IHC only. Cox and colleagues have shown that these are two distinct pathologic processes with different prognostic implications. ${ }^{4}$ The authors may have had no choice but to combine the two groups in order to provide enough patients to give their study adequate power.

The fact that most (25 of 27) of the micrometastasis were missed on frozen section is not surprising and 
parallels our experience. In our study evaluating scrape cytology for intraoperative assessment of SLNs, 35\% of patients with negative SLNs intraoperatively were found to have micrometastasis by serial H\&E staining. Twenty percent of the patients who went on to completion ALND were found to have additional positive lymph nodes. In the patients who elected against completion, there have been no axillary or distant recurrences. ${ }^{8}$

There are some drawbacks to this study, which the authors have acknowledged. The numbers are low, and follow-up in some patients was as short as 24 months, which is not long enough for evaluating recurrence or survival. Although the authors state that their patients were comparable in size, grade, location, and receptor status of the tumor, as well as with chemotherapy and hormone therapy regimens, some key differences are apparent. They discuss a standard protocol where patients with micrometastatic disease were treated the same as node-negative patients. However, three times as many node-positive patients received combined hormone and systemic adjuvant therapy, and they were more likely to receive adjuvant therapy in general than node-negative patients (96\% vs. $83 \%$.) This could affect the disease-free and overall survival findings in this study.

The detection of micrometastasis in axillary lymph nodes has steadily increased with the establishment of SLN biopsy as the standard of care for staging the axilla. In some studies, the incidence is reported to be as high as $23 \%$ of patients with invasive breast cancer. ${ }^{7}$ A likely contributing factor is that pathologists are able to focus on fewer nodes, thus allowing a more detailed examination. ${ }^{7}$ In addition, the increasing use of IHC staining for H\&Enegative sentinel nodes results in further detection of micrometastatic disease. ${ }^{2,9}$

With the increased detection of micrometastatic disease, our management of such patients is becoming more important. The larger numbers in the ACOSOG Z010 and Z0010 trials will, we hope, allow for further subgroup analysis to better account for confounding factors such as lymphovascular invasion and tumor grade, as well as separate the $\mathrm{pN}_{1 \text { mic }}$ from the $\mathrm{pNO}_{\mathrm{i}+}$ patients. This would certainly provide more reassurance to surgeons who are considering avoiding ALND in patients with positive SLNs. So unfortunately, the answer to the question about whether ALND can safely be omitted for all early-stage patients with micrometastasis is, we don't know yet, but we hope so.

\section{REFERENCES}

1. Tousimis E, Van Zee KJ, Fey JV, Hoque L, Tan LK, Cody HS, et al. The accuracy of sentinel lymph node biopsy in multicentric and multifocal invasive breast cancers. $J$ Am Coll Surg. 2003;197:529-35.

2. Gobardhan PD, Elias SG, Madsen EVE, Bongers V, Ruitenberg HJM, Perre CI, et al. Prognostic value of micrometastases in sentinel lymph nodes of patients with breast carcinoma: a cohort study. Ann Oncol. 2009;20:41-8.

3. Colleoni M, Rotmensz N, Peruzzotti G, Maisonneuve P, Mazzarol G, Pruneri G, et al. Size of breast cancer metastases in axillary lymph nodes: clinical relevance of minimal lymph node involvement. J Clin Oncol. 2005;23:1379-89.

4. Cox CE, Kiluk JV, Riker AI, Cox JM, Allred N, Ramos DC, et al. Significance of sentinel lymph node micrometastases in human breast cancer. J Am Coll Surg. 2007;206:261-8.

5. Dabbs DJ, Fung M, Landsittel D, McManus K, Johnson R. Sentinel lymph node micrometastasis as a predictor of axillary tumor burden. Breast J. 2004;10:101-5.

6. Colpaert C, Vermeulen P, Jeuris W, Van Beest P, Goovaerts G, Weyler J, et al. Early distant relapse in node-negative breast cancer patients is not predicted by occult axillary lymph node metastases, but by the features of the primary tumour. J Pathol. 2001;139: 442-9.

7. Cronin-Fenton DP, Ries LA, Clegg LX, Edwards BK. Rising incidence rates of breast carcinoma with micrometastatic lymph node involvement. J Natl Cancer Inst. 2007;99:1044-9.

8. Teal CB, Tabbara S, Kelly T. Evaluation of intraoperative scrape cytology for sentinel lymph node biopsy for patients with breast cancer. Breast J. 2007;13:155-7.

9. Giuliano AE, Dale PS, Turner RR, Morton DL, Evans SW, Krasne DL, et al. Improved axillary staging of breast cancer with sentinel lymphadenectomy. Ann Surg. 1995;222:394-9. 